\title{
Clinical Trial on the Incidence of Wound Infection and Patient Satisfaction After Stoma Closure: Comparison of Two Skin Closure Techniques
}

\author{
Sang Il Yoon, Sun Mi Bae, Hwan Namgung, Dong Guk Park \\ Department of Surgery, Dankook University College of Medicine, Cheonan, Korea
}

Purpose: Surgical site infection (SSI) is one of the most common complications that can occur after stoma closure. Reports have described differences in the incidence of wound infection depending on the skin closure technique, but there is no consensus on the ideal closure technique for a stoma wound. The aim of this study was to compare the incidence of SSI and the patient satisfaction between a circumferential purse-string approximation (CPA) and a primary linear closure (PC) of a stoma wound.

Methods: This prospective nonrandomized trial enrolled 48 patients who underwent a stoma closure from February 2010 to October 2013. Patients were divided into two groups according to the stoma closing technique: the CPA group $(\mathrm{n}=34)$ and the PC group $(n=14)$. The incidences of SSI for the two groups were compared, and the patients' satisfaction with the stoma closure was determined by using a questionnaire.

Results: SSI occurred in 3 of 48 patients (6.3\%) and was more frequent in the PC group than in the CPA group (3/14 [21.4\%] vs. $0 / 34$ [0\%], $\mathrm{P}=0.021$ ). Time to complete healing after stoma closure in the CPA group was 32 days (range, 1461 days). Patients in the CPA group were more satisfied with the resulting wound scar $(\mathrm{P}=0.043)$.

Conclusion: After stoma closure, CPA was associated with a significantly lower incidence of wound infection and greater patient satisfaction compared to PC. However, with the CPA technique, the time to heal is longer than it is with PC.

\section{Keywords: Surgical stomas; Wound closure technique; Wound infection; Patient satisfaction}

\section{INTRODUCTION}

A temporary stoma is frequently used in the treatment of colorectal cancer, inflammatory bowel disease and diverticulitis. In particular, it is used to reduce the anastomotic leakage and the reoperation rate upon low anterior resection of rectal cancer. Various complications can occur after stoma closure. Wound infection, small bowel obstruction, anastomotic leakage and incisional her-

Received: November 17, 2014 - Accepted: January 20, 2015

Correspondence to: Hwan Namgung, M.D.

Departments of Surgery, Dankook University College of Medicine,

119 Dandae-ro, Dongnam-gu, Cheonan 330-997, Korea

Tel: +82-41-550-3929, Fax: +82-41-556-3878

E-mail: gsnamgung@dankook.ac.kr

(C) 2015 The Korean Society of Coloproctology

This is an open-access article distributed under the terms of the Creative Commons Attribution NonCommercial License (http://creativecommons.org/licenses/by-nc/3.0) which permits unrestricted non-

commercial use, distribution, and reproduction in any medium, provided the original work is properly cited. nia are examples of complications. Among them, surgical site infection (SSI) is the most common complication, occurring in $2 \%-$ $40 \%$ of cases after primary linear closure (PC) $[1,2]$. Due to severe wound infection, prolonged hospital stay, medications, or outpatient wound care may be required. In addition, more time may be required before a normal life can be resumed.

PC of skin has been mainly used in stoma closure. However, Banerjee [3] introduced the circumferential purse-string approximation (CPA), reporting that after an ileostomy reversal, if wounds are closed by using purse-string skin closure, wound infection is less, and scars become smaller and are cosmetically superior. A prospective study reported that no SSI occurred in 51 patients who underwent a purse-string approximation [4]. After stoma closure, patient satisfaction and the cosmetic appearance of the wound scar are important, and studies evaluating these issues are underway. A meta-analysis of studies of cosmesis as a long-term outcome reported no significant difference between the PC and the CPA groups [5]. The purpose of this study is to analyze the difference in 
SSI rate between the PC group and the CPA group upon stoma closure and to discuss the differences in patient satisfaction with wound healing.

\section{METHODS}

Forty-eight patients who underwent stoma closure at Dankook University Hospital from February 2010 to October 2013 were enrolled. Two colorectal surgeons participated in this prospective study. According to the preference of the surgeon, skin closure was performed either by using a PC or a CPA. For the 48 patients, demographic data, premedical history, indications for stoma creation, stoma type, and operation time were determined to examine their relevance to wound infection. All patients underwent surgery under general anesthesia. Prophylactic antibiotics (first generation cephalosporin) were administered one hour before surgery. In the PC group, the stoma was mobilized from the abdominal wall through dissection after an elliptical skin incision. The protruding bowel was resected, and a side-to-side anastomosis was performed by using staplers. The fascia at the stoma site was closed with an absorbable multifilament interrupted suture. The peristomal incision was closed with staples or was sutured by using a nonabsorbable monofilament material (Fig. 1). No wound drainage was ap-
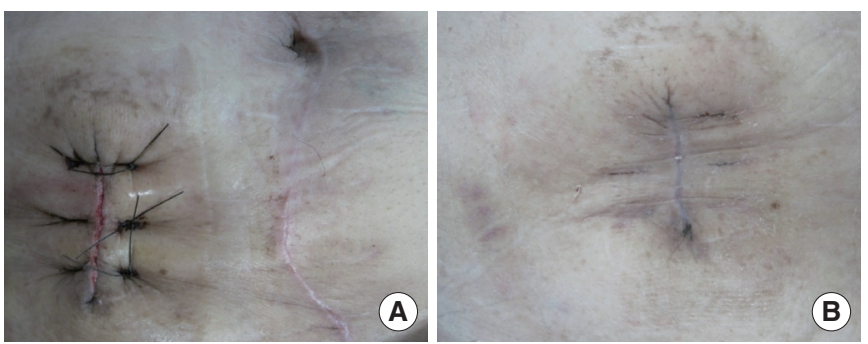

Fig. 1. (A) Primary linear closure wound on postoperative day 2 in a 73-year-old male patient. (B) Appearance of a scar after complete wound healing.
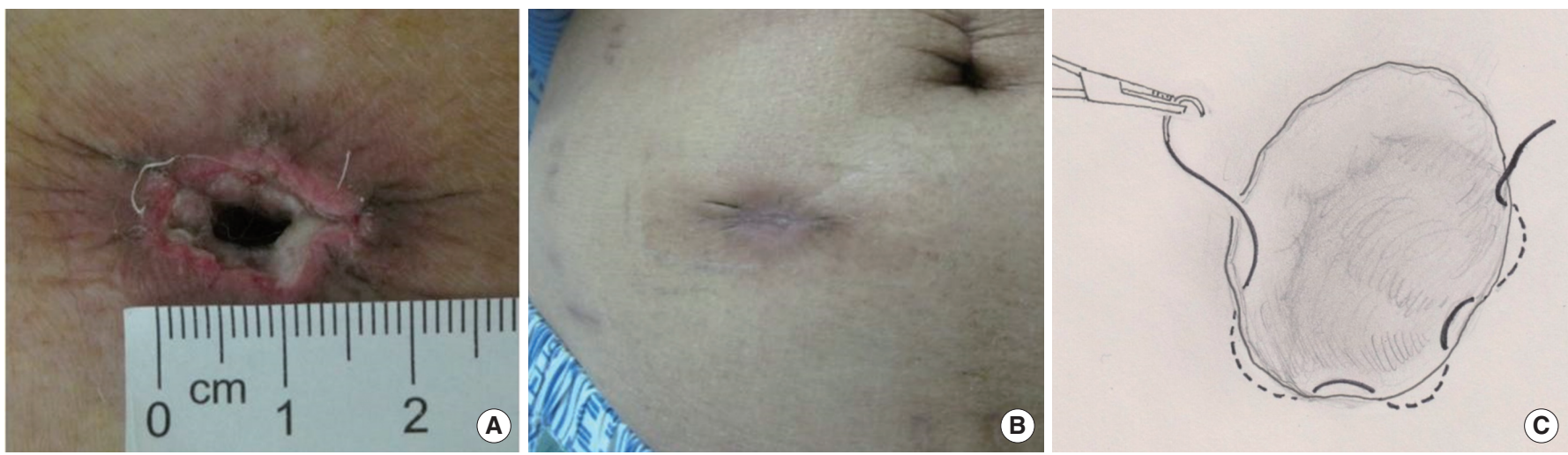

Fig. 2. (A) Circumferential purse-string approximation wound on postoperative day 2 in a 55-year-old male patient. (B) Appearance of a scar after complete wound healing. (C) Illustration showing the operative procedure for the circumferential purse-string approximation. plied for any of the patients who underwent a PC. After the completion of surgery, a simple sterile dressing was applied. In the CPA group, the peristomal incision was circular at the mucocutaneous junction. The bowel anastomosis and the fascial closure were the same as those in the PC group. A subcuticular suture was performed with the CPA method by using an absorbable 3-0 monofilament (Fig. 2). After the purse-string suture, a skin defect formed with a diameter of $1.5-2.0 \mathrm{~cm}$, and a sterile dressing was applied using a loose packing of povidone-iodine gauze.

SSI was evident by the presence of pus or dirty discharge, erythema, or induration, which is in accord with the standard of the Centers for Disease Control. Infected wounds were all opened and packed with gauze daily, and the skin was closed when evidence of wound infection no longer existed. The time to complete healing was defined as the time at which a dressing was no longer needed after stitch removal and the patient no longer needed medication for wound discomfort. In addition, a questionnaire was used to quantify and compare the differences in patient-reported woundhealing satisfaction and cosmesis. The Korean version of patient wound healing satisfaction scale was used after complete wound healing [6]. This scale is a 5-point Likert-type scale. A score of 1 to 5 was assigned for each factor, with higher scores indicating increased satisfaction. Factors assessed included patient satisfaction with appearance of the scar, expectations regarding the scar, level of postoperative pain, time of wound healing, difficulty of wound care and limitation of activity.

Statistical analyses were done using the PASW Statistics ver. 18.0 (SPSS Inc., Chicago, IL, USA). For continuous covariates, means were compared by using the Mann-Whitney U-test. Categorical variables were compared using the Fisher exact test for multiple outcomes. An effect was considered as statistically significant at $\mathrm{P}<0.05$.

\section{RESULTS}

We identified 48 patients (mean age, 58 years; range, 29-78 years; 16 females [33\%]) who underwent stoma closure during the study 
period of this study (PC, $\mathrm{n}=14 ; \mathrm{CPA}, \mathrm{n}=34$ ). There were no statistical differences in demographics, indications for stoma creation, and types of stoma between the two groups (Table 1). In addition, variations related to stoma closure were not statistically significant, except for the healing time. Time to complete healing in the PC group was shorter than it was in the CPA group (18.6 days vs. 32.1 days, $\mathrm{P}=0.000)$, and it was much shorter in the PC group when SSI cases were excluded (14.7 days). SSI occurred in three patients of the PC group, but did not occur in the CPA group $(\mathrm{P}=0.021)$ (Table 2).

All 48 patients completed a questionnaire concerning patient satisfaction and cosmesis. The mean time interval was 8 weeks ( 4 to 17 weeks) between ileostomy closure and completion of the questionnaire. The total score was higher in the CPA group. The CPA group showed a significantly higher score for expectations regarding the scar $(\mathrm{P}=0.043)$. There were no differences in the patients' satisfaction with the appearance of the scar, the level of postoperative pain, the time to wound healing, the difficulty of wound care and limitations on activities between the two groups (Fig. 3).

\section{DISCUSSION}

After stoma closure, complications, including small bowel obstruction, anastomotic leakage, and postincisional hernia, may occur. SSI is the most common complication. Depending on the author and the closure technique, the SSI rate varies from $0 \%$ to $41 \%$. Primary linear closure, second delayed closure, and purse-string closure have been introduced as stoma closure techniques, but there is no consensus on the ideal closure technique for a stoma wound despite continuing study. In this prospective nonrandomized trial, the SSI rate was $21.4 \%$ after primary linear closure, and no SSI occurred in the CPA group. Previous studies showed similar results [7-9].

There are many theories concerning why wound infection occurs less frequently following a CPA than following a PC. The advantage of the CPA is that, until granulation tissues grow and the skin is epithelialized, small skin defect areas become natural drainage pathways, which prevents wound infection [10]. Factors accounting for the high rate of infection after primary linear closure of the wound are unknown, although bacterial contamination of

Table 1. Patiens' characteristics

\begin{tabular}{|c|c|c|c|}
\hline Variable & $\begin{array}{l}\text { Circumferential purse-string } \\
\text { approximation }(n=34)\end{array}$ & $\begin{array}{l}\text { Primary linear } \\
\text { closure }(n=14)\end{array}$ & P-value \\
\hline Age (yr), mean (range) & $57.91(32-78)$ & $58.35(29-76)$ & 0.785 \\
\hline Gender & & & 0.328 \\
\hline Male:female & $21: 13$ & $11: 3$ & \\
\hline Body mass index $\left(\mathrm{kg} / \mathrm{m}^{2}\right)$ & 22.65 & 21.42 & 0.14 \\
\hline Diabetes mellitus & 5 & 3 & 0.676 \\
\hline Type of stoma & & & 0.588 \\
\hline Loop ileostomy & 24 & 12 & \\
\hline Transverse loop colostomy & 1 & 0 & \\
\hline Sigmoid loop colostomy & 4 & 2 & \\
\hline End colostomy & 3 & 0 & \\
\hline Other & 2 & 0 & \\
\hline Diagnosis & & & 0.76 \\
\hline Colo-rectal cancer & 22 & 10 & \\
\hline Inflammatory bowel disease & 2 & 0 & \\
\hline Trauma & 4 & 1 & \\
\hline Other & 6 & 3 & \\
\hline
\end{tabular}

Table 2. Variations related to stoma closure

\begin{tabular}{lccc}
\hline Variable & $\begin{array}{c}\text { Circumferential purse-string } \\
\text { approximation }(\mathrm{n}=34)\end{array}$ & $\begin{array}{c}\text { Primary linear } \\
\text { closure }(\mathrm{n}=14)\end{array}$ & P-value \\
\hline Mean time from stoma creation to closure (day) & 96.97 & 99.64 & 0.617 \\
Mean hospital day (day) & 16.44 & 14.79 & 0.64 \\
Mean operative time (min) & 134.94 & 106.07 & 0.022 \\
Time to complete healing (day) & 32.06 & 18.57 & $<0.001$ \\
Surgical site infection & 0 & 3 & 0.021 \\
\hline
\end{tabular}




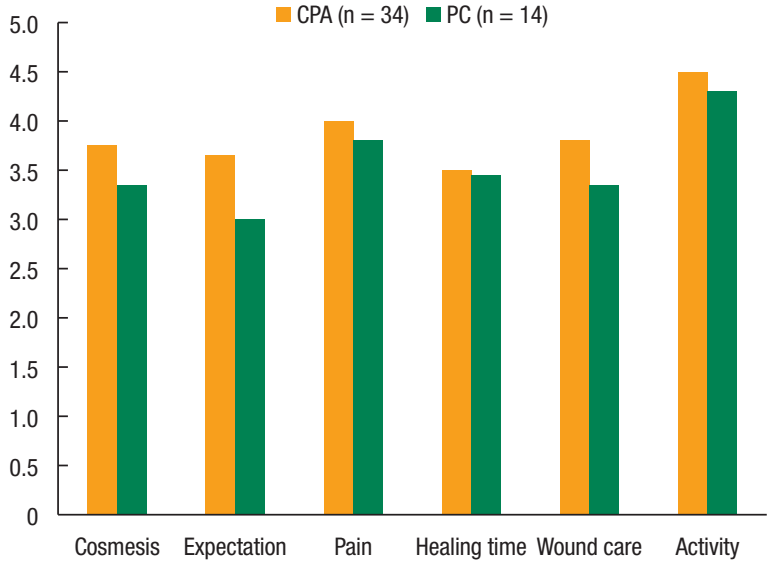

Fig. 3. Satisfactions scores for the wounds. CPA, circumferential pursestring approximation; PC, primary linear closure.

the peristomal skin probably plays an important role [6]. Concerning factors affecting wound infection, patients with higher body mass index and those whose anastomosis was hand-sewn could be at higher risk of SSI [11, 12]. Mileski et al. [13] reported that in 93 patients who received an ileostomy and colostomy reversal, hypertension and smoking were risk factors for wound infection. Akiyoshi et al. [14] reported that in 125 patients for whom an ileostomy had been performed after surgery for the treatment of rectal cancer, being male and having wound infection at the time of rectal cancer surgery were significant risk factors for wound infection after the ileostomy reversal. A study reported that significant risk factors for SSI were long operative time, increased blood loss and chronic renal failure in the PC group [12].

In our study, there was a difference in the time to complete healing between the PC and the CPA groups. Williams et al. [15] expressed concern about a longer wound-healing period and the increased medical cost in cases involving purse-string suture as discharge was drained continuously through the skin. In contrast to our findings, a prior study reported that the wound-healing period of linear skin closure was 24.6 days and that of purse-string skin closure was 20.6 days, but the difference was not statistically significant [8]. Camacho-Mauries et al. [16] reported that a subgroup of patients who did not have an infection had a shorter healing time of 2 weeks, which is approximately half the healing time in the purse-string group. With the purse-string procedure, keeping the wound clean at home by washing it with soap and water is a must. The patient does not need to go to the hospital to receive any special treatment. Although no significant differences were noted in the present study, patients' responses indicated that the CPA was associated with easier management of the dressing and less discomfort.

According to the results of the questionnaire, CPA patients reported higher scores for all the questions. However, the only statistical difference was expectations regarding the scar $(\mathrm{P}=0.044)$.
A previous study reported a trend toward better cosmetic results for circumferential subcuticular wound approximation than for PC [6]. Another study of patients who underwent a CPA found that while the initial circular scar might be unappealing, final scar formation occurred along natural skin tension lines, producing a cosmetically pleasant scar [17]. Milanchi et al. [6] reported a significantly higher mean patient satisfaction score in the circumferential subcuticular wound approximation group than in the PC group.

This study has several limitations. The study was nonrandomized, and the number of patients was small. Finally, the SSI rate in the PC group was higher than it was in other studies for reasons that are unclear. Although the SSI rate was high, considering the small case number, we think that prophylactic antibiotics might have influenced the incidence of SSI. Thus, we are considering a change of prophylactic antibiotics.

If the CPA was performed as a skin closure technique after stoma reversal, the incidence of SSI was lower than it was when a PC was used. Also, patient satisfaction with the wound scar was greater in the CPA group than in the PC group. However, the CPA technique takes a longer time to heal than the PC technique. We think that CPA may be a good alternative option, but further prospective randomized trials involving more patients are necessary before a definitive conclusion can be drawn.

\section{CONFLICT OF INTEREST}

No potential conflict of interest relevant to this article was reported.

\section{ACKNOWLEDGMENTS}

We are indebted to Young Sun Ju for good illustrations.

\section{REFERENCES}

1. Wong KS, Remzi FH, Gorgun E, Arrigain S, Church JM, Preen M, et al. Loop ileostomy closure after restorative proctocolectomy: outcome in 1,504 patients. Dis Colon Rectum 2005;48:243-50.

2. Hackam DJ, Rotstein OD. Stoma closure and wound infection: an evaluation of risk factors. Can J Surg 1995;38:144-8.

3. Banerjee A. Pursestring skin closure after stoma reversal. Dis Colon Rectum 1997;40:993-4.

4. Sutton CD, Williams N, Marshall LJ, Lloyd G, Thomas WM. A technique for wound closure that minimizes sepsis after stoma closure. ANZ J Surg 2002;72:766-7.

5. McCartan DP, Burke JP, Walsh SR, Coffey JC. Purse-string approximation is superior to primary skin closure following stoma reversal: a systematic review and meta-analysis. Tech Coloproctol 2013; 17:345-51.

6. Milanchi S, Nasseri Y, Kidner T, Fleshner P. Wound infection after ileostomy closure can be eliminated by circumferential subcu- 
ticular wound approximation. Dis Colon Rectum 2009;52:469-74.

7. Pittman DM, Smith LE. Complications of colostomy closure. Dis Colon Rectum 1985;28:836-43.

8. Reid K, Pockney P, Pollitt T, Draganic B, Smith SR. Randomized clinical trial of short-term outcomes following purse-string versus conventional closure of ileostomy wounds. Br J Surg 2010;97:15117.

9. Parks SE, Hastings PR. Complications of colostomy closure. Am J Surg 1985;149:672-5.

10. Lee JR, Kim YW, Sung JJ, Song OP, Kim HC, Lim CW, et al. Conventional linear versus purse-string skin closure after loop ileostomy reversal: comparison of wound infection rates and operative outcomes. J Korean Soc Coloproctol 2011;27:58-63.

11. Konishi T, Watanabe T, Kishimoto J, Nagawa H. Elective colon and rectal surgery differ in risk factors for wound infection: results of prospective surveillance. Ann Surg 2006;244:758-63.

12. Marquez TT, Christoforidis D, Abraham A, Madoff RD, Rothenberger DA. Wound infection following stoma takedown: primary skin closure versus subcuticular purse-string suture. World J Surg
2010;34:2877-82.

13. Mileski WJ, Rege RV, Joehl RJ, Nahrwold DL. Rates of morbidity and mortality after closure of loop and end colostomy. Surg Gynecol Obstet 1990;171:17-21.

14. Akiyoshi T, Fujimoto Y, Konishi T, Kuroyanagi H, Ueno M, Oya M, et al. Complications of loop ileostomy closure in patients with rectal tumor. World J Surg 2010;34:1937-42.

15. Williams LA, Sagar PM, Finan PJ, Burke D. The outcome of loop ileostomy closure: a prospective study. Colorectal Dis 2008;10:4604.

16. Camacho-Mauries D, Rodriguez-Diaz JL, Salgado-Nesme N, Gonzalez QH, Vergara-Fernandez O. Randomized clinical trial of intestinal ostomy takedown comparing pursestring wound closure vs conventional closure to eliminate the risk of wound infection. Dis Colon Rectum 2013;56:205-11.

17. Klink CD, Wunschmann M, Binnebosel M, Alizai HP, Lambertz A, Boehm G, et al. Influence of skin closure technique on surgical site infection after loop ileostomy reversal: retrospective cohort study. Int J Surg 2013;11:1123-5. 